\title{
Las andanzas laborales de las pioneras de la farmacia en Guadalajara
}

\author{
The labor trajectories of \\ the pharmaceutical women in Guadalajara
}

\author{
Luciano Oropeza Sandoval \\ Departamento de Estudios en Educación, \\ Universidad de Guadalajara \\ loropezasandoval@yahoo.com.mx
}

\begin{abstract}
Resumen
En este escrito, se examina la trayectoria laboral que trazaron las primeras mujeres que estudiaron la carrera de farmacia en Guadalajara; asimismo, se describe el tipo de actividades que realizaron, las condiciones de empleo que existieron para las egresadas de esa carrera y las acciones que emprendieron para crear opciones más acordes con su condición femenina. Este estudio abarca desde 1900, año en el que egresó la primera generación farmacéutica, hasta principios de 1940, década en que el grupo estudiado culminó prácticamente su vida laboral activa.

Este artículo revela que las farmacéuticas establecieron boticas, donde se desempeñaron como propietarias y profesoras a cargo del despacho de medicinas; también muestra que dos de ellas alternaron esta labor con el trabajo de enseñanza en escuelas de formación femenina. Estas actividades les permitieron materializar sus expectativas laborales, pero también afectaron su trayectoria personal, porque el modelo de la mujer trabajadora no era compatible con el ideal de la mujer casadera.
\end{abstract}

Palabras clave: farmacia, mujeres y trayectoria laboral, Guadalajara.

\section{Abstract}

This paper examines the career trajectory traced by the first women studying the pharmacy career in Cuadalajara. Here we describe the type of activities they perform, the conditions of employment 
that exist for the graduates of that career and the actions they undertake to create options more in line with their female status. This study covers from 1900, the year in which the first pharmacist graduated, until the beginning of 1940, a decade in which the group studied practically closes his active working life.

This study reveals that pharmacists manage to establish pharmacies where they work as owners and teachers in charge of dispensing medicines. It also shows that two of them alternate this activity with teaching work in women's training schools. These activities allow them to materialize their labor expectations, but also affect their personal trajectory, because the model of the working woman is not compatible with the ideal of married women. They, like many normalist teachers, remain single throughout their life.

Keywords: Pharmacy, Women and Career Trayectory, Guadalajara.

\section{Introducción}

En este escrito, se examina la trayectoria laboral que trazaron las primeras mujeres que estudiaron la carrera de farmacia en Guadalajara; ${ }^{1}$ asimismo, se describe el tipo de actividades laborales que realizaron, las condiciones de empleo que existieron para las egresadas de esa carrera y las acciones que emprendieron para crear opciones acordes con las ideas de recato y honorabilidad vigentes en torno al trabajo femenino. Estas primeras farmacéuticas no desarrollaron actividades distintas a las que realizaron sus pares varones, pero sí desplegaron acciones que les permitieron liberarse de la afrenta que se les endosó por convivir con los hombres en espacios públicos.

Este estudio abarca desde 1900, año en el que egresó la primera farmacéutica, hasta principios de 1940, década en que el grupo estudiado culminó prácticamente su vida laboral activa; se basa en información proveniente del Archivo Histórico de la Universidad de Guadalajara, del Archivo Histórico de Jalisco, del Archivo de Instrucción Pública, del Archivo Histórico de la Secretaría de Salud y de fondos hemerográicos, especialmente, del periódico El Informador.

Para conocer la trayectoria laboral de las profesoras de farmacia, recurrimos a la prosopografía, estrategia que permite acopiar información relacionada con sus actividades remu-

\footnotetext{
${ }^{1}$ Este escrito forma parte de un proyecto de investigación denominado "La trayectoria laboral de las profesoras de Farmacia en Guadalajara: 1900-1950", donde se examinan las actividades remunerativas que realizaron tanto las egresadas de la carrera de farmacia de la Facultad de Medicina y Farmacia, como las mujeres que terminaron la carrera análoga en la Escuela Comercial e Industrial para Señoritas (ECIS). Aquí sólo se examina la trayectoria laboral de las primeras farmacéuticas, motivo por el cual no se describen con detenimiento las particularidades educativas de la ECIS, porque estas mujeres no egresaron de esta institución. En un ensayo subsecuente, donde se aborda el recorrido de las egresadas de farmacia de la ECIs, sí se incluye una descripción detallada de este proyecto escolar.
} 
nerativas y sociales (Stone, 1986). Esta capacidad empírica que ofrece esta opción metodológica se combinó con las aportaciones generadas desde la metodología biográfica, enfoque que hace énfasis en el contexto y el sujeto biografiado (Bazant, 2013). Acorde con esta conjunción de estrategias, se cree que el contexto es un nivel de realidad importante para entender dónde y cómo esas mujeres lograron desarrollar proyectos individuales mediante los cuales superaron las expectativas laborales y sociales establecidas para el género femenino en las primeras décadas del siglo xx en Guadalajara.

En ese sentido, el contexto incluye, no sólo las posibilidades objetivas de empleo, sino también las condiciones socioculturales que influyeron en el acceso de estas mujeres al trabajo remunerativo. En esta lógica, se utilizó la prosopografía como una estrategia metodológica que busca acopiar información vinculada tanto con las condiciones materiales y socioculturales que rodearon al trabajo profesional de la farmacia, en especial al trabajo femenino, como con las acciones que desarrollaron las egresadas de esta carrera para acceder a los espacios laborales.

A principios del siglo xx, la actividad del profesor de farmacia se limitaba básicamente al despacho de medicinas en una botica. Esta reducida labor se amplió por las posibilidades socioeconómicas, por el reconocimiento a su papel profesional y por las relaciones que tuvieron con los grupos políticos locales y con las clases sociales de la localidad; sin embargo, estas oportunidades no se presentaron por igual para los egresados de esta carrera. Así, se puede decir que su trayectoria se caracterizó por la existencia de pocas opciones de desarrollo laboral. En un lado, se ubicaban los farmacéuticos con recursos socioeconómicos para establecer una farmacia y los profesores que se limitaban a contratar sus servicios para atender el despacho de medicinas, requsito indispensable para autorizar la apertura de una botica; en otro, los farmacéuticos médicos que, a la par de ser propietarios de farmacias ofrecían consulta en espacios anexos a esos establecimientos, y los farmacéuticos que tenían mayor reconocimiento, quienes alternaban alguna de las anteriores actividades con el ejercicio de labores docentes en la carrera de farmacia, o formaron parte del cuerpo directivo del Consejo Superior de Salubridad.

Estas opciones fueron más estrechas para las mujeres, quienes no sólo afrontaron las limitaciones derivadas de su situación socioeconómica, sino también las restricciones socioculturales existentes en torno a su vinculación con espacios asociados a la representación popular o al desempeño de puestos jerárquicos en órganos vinculados con la higiene y la medicalización. En ese orden, se postula que la generación pionera de farmacéuticas realizó, principalmente, el despacho de medicinas, actividad que, en algunos casos, alternó con labores de enseñanza. 


\section{El acceso de las mujeres a los espacios públicos en Guadalajara}

Para entender cómo se creó la profesión de farmacia para las mujeres tapatías, se narran algunos sucesos históricos que permiten ver cómo se abrieron nuevos segmentos de la vida pública para este grupo social; asimismo, se refieren tanto aspectos relacionados con la escolarización media femenina, como con el desarrollo económico regional y la demanda de fuerza de trabajo. Con estos datos, se pretende explicar el escenario que hizo posible el ingreso de las mujeres a esa carrera y al ámbito laboral de la farmacia.

La primera opción de educación media femenina surgió con la creación del Liceo de Niñas, organismo que se inauguró el 15 de octubre de 1861. Con esta escuela, se pretendió formar un ideal de mujer acorde con las características socioculturales dominantes en ese tiempo, es decir, una con mayor cultura, pero virtuosa y hábil para desempeñar las labores del hogar. En este plan de estudios inicial, coexistían tanto contenidos de enseñanza religiosa, como aspectos relacionados con las ideas de progreso social que enarbolaban los grupos liberales.

En este programa se expresan saberes que no sólo se relacionan con los grupos liberales, sino también con la fuerte influencia que ejerce la iglesia en torno a la vida social. Es decir, el plan curricular se estructura a partir de las concepciones predominantes sobre la virtud femenina y el progreso social. Las concepciones que hay en torno a la mujer están fuertemente permeadas por la Iglesia católica y son compartidas por conservadores y liberales. Estos pensamientos se expresan a través de la enseñanza religiosa y la moral.

Junto a estas concepciones sobre la virtud de la mujer aparecen conocimientos en torno al progreso económico y social. En ese entonces la idea de progreso se relaciona con un desarrollo de habilidades más sólidas relacionadas con la organización familiar. Por ejemplo, hay algunas materias que alientan el desarrollo de ciertas actividades laborales de las mujeres, como sucede con Economía doméstica y las labores de aguja, pero son saberes que fundamentalmente buscan mejorar las capacidades femeninas en torno a la administración económica del hogar (Figueroa, 2009: 48-49).

Esta propuesta de enseñanza no capacitó a las mujeres para realizar un trabajo externo al hogar. Sus posibilidades educativas se quedaron en el nivel de formación que otorgaba el Liceo de Niñas, debido a que el plan de estudios no constituía un antecedente escolar previo para ingresar a las carreras profesionales liberales, como sí sucedía con el Liceo de Varones (Peregrina, 2006). Esta limitación se relacionaba con una de las representaciones sociales en boga: que las mujeres no eran aptas para desempeñar las profesiones liberales. 
Lo anterior no significaba que las posibilidades de crecimiento social de las alumnas se quedaran circunscritas a la conclusión de los estudios formales del Liceo de Niñas, ya que al seno de este lugar se generaban experiencias culturales que incentivaban afinidades femeninas hacia la enseñanza; es decir, aunque curricularmente no se especificaba ninguna materia que instruyera en torno a ese oficio, había actividades cotidianas que involucraban a las mujeres en esa labor². Estas circunstancias, relacionadas con la demanda de mayor número de profesores y la creciente aceptación de miembros del género femenino en espacios de trabajo relacionados con la instrucción, ${ }^{3}$ hicieron posible que algunas de las egresadas se vincularan a la enseñanza como una actividad laboral.

Estos procesos tendieron a oficializarse en 1870, en ese año, el gobernador, Antonio Gómez Cuervo, ordenó a la Junta Directiva de Estudios que premiara a las alumnas destacadas con un título de preceptoras, impulsándolas a que impartieran algunas asignaturas en el plantel (Núñez, 1994); esta decisión es una prueba de la aceptación tácita de la creciente incorporación de la mujer al ámbito de la enseñanza. En esa época, se ampliaron las escuelas de niños y niñas, y se desató una mayor circulación de ideas políticas que fomentaron la educación femenina y justificaron la integración de las mujeres al trabajo de enseñanza, ${ }^{4}$ por considerar a éste como una prolongación de las tareas domésticas (Fernández, 1995).

Para 1879, al calor de la influencia del positivismo en la vida social de Guadalajara, se en el plan de estudios del Liceo de Niñas, se añadieron asignaturas que incluyeron contenidos para mejorar el trabajo en el hogar, para ampliar la formación cultural y para asuntos financieros básicos. ${ }^{5}$ Este cambio introdujo más espacios de acción para las mujeres, porque

2 En el Reglamento Interior del Liceo de Niñas de 1878, se estipulaba que la rectora podía "nombrar a las alumnas más adelantadas para que suplan las faltas accidentales de los profesores, siempre que estas no excedan de ocho días" [Archivo Histórico de la Universidad de Guadalajara (en adelante AHUC), Colección de los Decretos, Circulares y Órdenes de los Poderes del estado de Jalisco, tomo VII, pp. 166-182]. Aunque la temporalidad de este reglamento no coincide exactamente en el tiempo con lo referido en el párrafo, se cree que estas normas evidencian la existencia de prácticas que se realizaron desde años atrás.

${ }^{3}$ García (2005) muestra cómo las preceptoras incrementaron su participación en la enseñanza en Jalisco. De 1856 a 1860, 31.42\% de los profesores se integraba por mujeres, proporción que, de 1872 a 1876, ascendió hasta llegar a $85.62 \%$.

${ }^{4}$ Peregrina (2006) y Alvarado (2004) señalan que su incorporación a la enseñanza se debió también a intereses de orden económico, puesto que las mujeres recibían una baja remuneración salarial, lo cual proporcionaba un gran ahorro monetario al gobierno. Por su parte, Cano (1996: 40) aduce que "los funcionarios públicos de esa época aceptaban que había motivos de carácter económico para considerar conveniente el trabajo femenino: una profesora resultaba 'más barata' que un profesor, esto porque el ingreso salarial de las mujeres era pensado como "una aportación complementaria, y no esencial, para el sostenimiento de una familia". Estos funcionarios reconocieron que las mujeres tenían mayor dedicación y permanencia en el trabajo de enseñanza, lo cual no ocurría con los hombres, quienes frecuentemente abandonaban el magisterio para desempeñarse en otros campos profesionales (Cano, 1996).

${ }^{5}$ Figueroa (2009: 70 y 71) dice que a la mujer "se le ilustraba en conocimientos teóricos de economía doméstica y se le inculcaba la moral de México moderno a través de los ramos de urbanidad, moral, historia de México e historia general; también se les dotaba de 'adornos culturales' como música, jardinería, botánica, litografía, labores manuales, etc. En esta formación de carácter elitista, se le abrieron las puertas de la información y la cultura, a través 
se aceptaba que ellas sí podían estudiar como los hombres, pero sólo aquellos saberes que se asociaban con los roles que realizaban en la casa. Así, el positivismo legitimó su incorporación a la educación superior, mas limitó su acceso a las opciones vinculadas a las labores consideradas "femeninas", como sucedió con la enseñanza.

En esta nueva organización, también se subrayaba la importancia de la virtud de la mujer, cualidad que se transmitía a través de las materias de urbanidad, moral y economía doméstica, y de la práctica que desarrollaban autoridades y profesores. En el Reglamento de 1879, se especificaba que la rectora debía supervisar que las alumnas cumplieran con sus deberes religiosos y que los profesores examinaran su presencia diaria, revisando que su comportamiento fuera conforme a "las maneras propias de una sociedad culta, [cuidando] muy especialmente de su moralidad". 6

En este plan de estudios, se evidenciaba que la acepción de la virtud femenina estaba fuertemente influida por las representaciones sociales que difundía la Iglesia católica, pero también aparecían ideas más tolerantes en torno a la participación de las mujeres en los espacios educativos, porque se argumentaba que podían asistir a la educación superior, siempre y cuando, su elección se orientara hacia carreras consideradas afines a su condición de género. Estas ideas discrepantes del conservadurismo católico provenían de los grupos más radicales del liberalismo local, sobre todo, de los vinculados con las tradiciones masónicas y protestantes.

En los años 80, persistía la percepción de que la educación femenina debía orientarse hacia la formación de una mujer virtuosa y a la generación de actividades laborales compatibles con su sexo, como puede verse en el Reglamento de la Instrucción Primaria del Estado de Jalisco, donde se estipulaba que los objetivos del Liceo de Niñas eran:

I. Inspirar a la mujer desde su niñez los sentimientos más nobles para que sea virtuosa en cualquier condición de vida, encaminándola a la perfección dentro de la órbita de la moral más pura.

II. Dedicarla al estudio para que pueda llegar a ejercer el preceptorado o alguna otra profesión compatible con su sexo.

III. Enseñarle las reglas de urbanidad. ${ }^{7}$

de la gramática, la literatura y la enseñanza de idiomas vivos como italiano, francés e inglés. Además, se deja entrever que la mujer podía ser productiva, desde el punto de vista económico, por tanto se le enseñaba teneduría de libros, aplicable en el hogar y en los negocios".

${ }^{6}$ Reglamento Interior del Liceo de Niñas aprobado por la Junta Directiva de Estudios del estado (Biblioteca Pública del Estado de Jalisco, miscelánea 733, documento 43).

${ }^{7}$ Reglamento para el Liceo de Niñas del Estado, decreto núm. 214, enero 10 de 1884 (Biblioteca Pública del Estado de Jalisco, educación media, exp. 301). 
Hasta 1887, la propuesta educativa del Liceo de Niñas no incluyó contenidos curriculares que capacitaran a las alumnas para el ejercicio de la enseñanza, ${ }^{8}$ mucho menos para realizar estudios vinculados con las profesiones liberales; sin embargo, dos años después, el gobernador, Ramón Corona, aprobó una nueva ley de instrucción pública donde se prescribía la creación de escuelas normales en esa institución educativa y en el Liceo de Varones, respectivamente. En ambos planteles se incluyeron las carreras de "preceptores de primer orden ó de instrucción primaria superior [y preceptores] de instrucción primaria elemental"; 9 en la primera, se anexó la asignatura de pedagogía en el quinto año, y la de instrucción primaria elemental, en el segundo y tercer año.

En esa misma ley, se ordenaba la adición de las cátedras de telegrafía, electrotécnica, telefonía, mecanografía y taquigrafía al plan de estudios del Liceo de Niñas; con estas asignaturas, correspondientes a las carreras de teneduría de libros y de telegrafista, se pretendía proveer a las alumnas de saberes y técnicas que les permitieran incursionar en actividades laborales relacionadas con los nuevos medios de comunicación, como el telégrafo y la telefonía, y con la Administración Pública y el comercio.

En 1894, hubo otra modificación curricular; el gobernador de Jalisco, Luis C. Curiel, promovió una reorganización de los dos liceos, el de Niñas y el de Varones, donde dispuso que esa instrucción sirviera de preparación para todas las carreras profesionales existentes, mientras se abría una preparatoria general. En el Liceo de Niñas, además de las carreras de telegrafista, normalista de instrucción primaria elemental y primaria superior (Mata, 1985), se ofreció la preparatoria para las alumnas que deseaban estudiar las profesiones de teneduría de libros y farmacia. ${ }^{10}$ Estos cambios en el plan de estudios no alteraron sus preferencias, ya que la mayoría eligió ser preceptora; en 1896, cursaban 64 estudiantes la carrera de profesora de instrucción primaria elemental; cinco, la de profesora de instrucción primaria superior; y 54, los estudios preparatorios para las carreras de teneduría de libros, telegrafista y farmacia. ${ }^{11}$

La apertura de estas opciones no implicó cambios socioculturales en torno al uso de los espacios públicos. Ellas asistieron a las escuelas de nivel superior investidas de los saberes que, convencionalmente, debían acompañar su comportamiento, hecho que las llevó a afrontar escenarios tormentosos. Por lo tanto, sus experiencias de formación se entremezclaron con los deseos de un gobierno que reivindicaba la participación de las mujeres en el

\footnotetext{
${ }^{8}$ Hay discrepancias en torno a la fecha de inclusión de la materia de pedagogía al Liceo de Niñas; mientras Cárdenas y Pío (1997) afirman que fue en 1887, Peregrina (2006) refiere que fue hasta 1889

${ }^{9}$ El 6 de junio de 1889, el gobernador, Ramón Corona, expidió el decreto 359 que dio vigencia a la Ley Orgánica de la Instrucción Pública (AHUG, Colección de los Decretos, Circulares y Órdenes de los Poderes del estado de Jalisco, tomo XII, pp. 398-343).

${ }^{10}$ Archivo Histórico de Jalisco (AHJ), IP-5-896 Gua/348.

${ }^{11}$ Archivo Histórico de Jalisco, IP-5-896 Gua/348; véase Figueroa, 2009: 91.
} 
desarrollo económico y social de la región, y con las creencias de una sociedad que les exigía tener una conducta que conservara sin tacha su virtud. Este paradójico ambiente sociocultural fue el que experimentaron las primeras alumnas de la carrera de farmacia, quienes implementaron estrategias de estudio para no compartir los espacios de la Facultad de Medicina y Farmacia con los estudiantes varones, acción que les permitió mantener sin menoscabo su reputación social (Oropeza, 2016).

\section{La inserción de las mujeres en el campo laboral de la farmacia}

Al igual que otras entidades de México, Jalisco experimentó un fuerte crecimiento económico en la segunda mitad del siglo xIX, en ese lapso surgieron fábricas textiles, cigarreras, numerosos comercios y oficinas especializadas en la provisión de diversos servicios. Esta expansión generó una ascendente demanda de fuerza de trabajo; en 1895, en Jalisco, la Población Económicamente Activa (PEA) ya sumaba 443709 trabajadores, de los cuales, $14.5 \%$ se conformaba por mujeres. En ese año, el personal femenino se concentraba en los ramos del comercio (29\%) y en la industria, las bellas artes y las artes y los oficios (55.6\%), destacando las mujeres vinculadas al servicio doméstico, las costureras, las lavanderas, tortilleras, las molenderas, las cigarreras, las obreras y las empleadas de establecimientos industriales (véase cuadro 1).

En la categoría relativa a las ocupaciones profesionales, las mujeres apenas representaban $2 \%$ de la PEA femenina; principalmente, se ubicaban en labores relacionadas con el magisterio y la partería, y en menor proporción, en la farmacia, la medicina y la Administración Pública (Dirección General de Estadística, 1899). Esta pequeña proporción de trabajadoras con mayor capacitación técnica, que aparece en los datos censales, permite ver cómo la diversificación de actividades económicas y sociales generó una demanda de fuerza de trabajo distinta a la PEA femenina ocupada en las actividades fabriles, en el comercio, en los servicios domésticos y en los oficios de abastecimiento básico.

El crecimiento de la educación elemental durante las primeras dos décadas del porfiriato, la modernización de las actividades comerciales y de servicios, así como el arribo del telégrafo y la telefonía demandaron mano de obra con capacitación técnica que no podía ser suministrada por las escuelas municipales, sino por instituciones de mayor escolaridad. Este requerimiento laboral fungió como un factor incidente en las reformas que se introdujeron en el Liceo de Niñas desde finales de los años 80, las cuales permitieron la apertura de carreras relacionadas con la telegrafía, la teneduría de libros y el trabajo farmacéutico. 


\section{Cuadro 1}

Principales ocupaciones de las mujeres en 1895

\begin{tabular}{|c|c|c|c|}
\hline Oficio u ocupación & $\begin{array}{c}\text { Hombres } \\
\%\end{array}$ & $\begin{array}{c}\text { Mujeres } \\
\%\end{array}$ & Total \\
\hline Servicios domésticos & $5942(25.9)$ & $16989(74.1)$ & 22931 \\
\hline Costureras & & $5798(100)$ & 5798 \\
\hline Comerciantes & $13065(70.9)$ & $5358(29.1)$ & 18423 \\
\hline Lavanderas & $10(00.2)$ & $4885(99.8)$ & 4895 \\
\hline Tortilleras & & $3624(100)$ & 3624 \\
\hline Molenderas & & $3438(100)$ & 3438 \\
\hline Propietarios & $2503(44.4)$ & $3133(55.6)$ & 5636 \\
\hline Tejedores & $3433(52.6)$ & $3096(47.4)$ & 529 \\
\hline Obreros de establecimientos industriales & $2117(56.3)$ & $1644(43.7)$ & 3761 \\
\hline Cigarreras & & $907(100)$ & 907 \\
\hline Magisterio & $529(38.6)$ & $836(61.4)$ & 1365 \\
\hline Alfareros & $3634(84.6)$ & $661(15.4)$ & 4295 \\
\hline Vendedores ambulantes & $564(48.7)$ & $594(51.3)$ & 1158 \\
\hline Sombrereros & $3013(85.2)$ & $522(14.8)$ & 3535 \\
\hline Obstetricia & & $265(100)$ & 265 \\
\hline $\begin{array}{l}\text { Administradores y empleados de } \\
\text { establecimientos industriales }\end{array}$ & $192(43.8)$ & $246(56.2)$ & 438 \\
\hline Mesalinas & & $239(100)$ & 239 \\
\hline Dulceros & $355(68.0)$ & $167(32.0)$ & 522 \\
\hline Empleados particulares & $878(87.8)$ & $122(12.2)$ & 1000 \\
\hline Empleados públicos & $1247(92.9)$ & $96(7.1)$ & 1343 \\
\hline Floristas & $15(14.0)$ & $92(86.0)$ & 107 \\
\hline Aparadores de Calzado & & $61(100)$ & 61 \\
\hline Bordadores & $3(5.6)$ & $51(94.4)$ & 54 \\
\hline
\end{tabular}

Fuente: Censo General de la república mexicana de 1895 (1899). Dirección General de Estadística, México.

Esta demanda de fuerza de trabajo femenina calificada enfrentó situaciones contradictorias porque, aunque la mayoría de las nuevas profesionistas realizó sus estudios en el Liceo de niñas, muchas de ellas no ejercieron actividades laborales asociadas con la capacitación recibida - las egresadas de farmacia constituyen una excepción, porque sí vincularon sus estudios con la práctica laboral de su profesión.

Para entender cómo estas mujeres consiguieron romper relativamente el monopolio que los hombres ejercían en torno a las carreras de nivel superior, es pertinente describir el esta- 
do que guardaba el ramo de las boticas y farmacias al inicio de los años 90 del siglo XIX. En esa década, la urbe tapatía contaba con 24 boticas; de las cuales, 15 eran propiedad de profesores de farmacia; cuatro, de médicos; y cinco, de particulares (Orendain, 1969).

En esa época, el Código Sanitario estipulaba que en cada botica debía haber un farmacéutico legalmente autorizado para atender el despacho de las medicinas; sin embargo, poco se respetaba esta prescripción, porque tanto profesores de farmacia como médicos y particulares incurrían en violaciones constantes de esa ley. En el caso de los farmacéuticos, había varios que no estaban titulados; y otros, que recurrían al uso de prácticos de farmacia, así como a personal que no tenía los estudios relacionados con esa disciplina y que eran formados por los mismos farmacéuticos - también los médicos y los particulares recurrían a este último para la elaboración y el despacho de medicamentos. Esta estrategia laboral les permitió reducir costos de mano de obra y les ayudó a aumentar sus utilidades, sin prever que estos hechos afectarían la imagen social de los profesores de farmacia.

La perspectiva laboral más interesante para los egresados de la carrera de farmacia era la instalación de una botica; sin embargo, esta opción requería de recursos económicos que no estaban al alcance de todos ellos. En esas circunstancias, varios optaron por ofrecer sus servicios a los propietarios de boticas y farmacias, quienes, al contar con la disposición de prácticos de farmacia, les ofrecían un salario poco atractivo para su estatus profesional; ante esta situación, muchos decidieron emigrar de la ciudad y ejercer en otras localidades, donde los requisitos y los recursos para instalar una farmacia eran menores.

Algunos profesores de farmacia, que sí operaban conforme a las prescripciones legales, denunciaron ante el Consejo Superior de Salubridad las irregularidades que existían en torno al despacho de medicinas.; exigieron a las autoridades mayor rigor en la aplicación del Código Sanitario, en contraparte, los propietarios de farmacia argumentaron que en la ciudad no existía personal especializado suficiente para hacerse cargo de esa labor y que por eso recurrían al uso de prácticos de farmacia.

La baja remuneración que se ofrecía a los farmacéuticos no sólo propició que muchos de ellos cambiaran de residencia, sino también afectó la imagen que la sociedad y los jóvenes tapatíos habían creado en torno a la profesión de farmacia. Esta menor apreciación se expresó en un creciente desinterés hacia los estudios de esa carrera; la población escolar de farmacia disminuyó a tal grado que, en algunos años de la década de los 90 no hubo ningún alumno inscrito. 12

En este ambiente de irregularidades y de devaluación de la imagen de los farmacéuticos, se gestó la permisibilidad de las autoridades y los miembros del gremio médico-farmacéutico para franquear el acceso de las mujeres al campo laboral de la farmacia. Con la capacitación

\footnotetext{
12 Archivo Histórico de la Universidad de Guadalajara (AHUG), Fondo Antecedentes Históricos de la Universidad de Guadalajara, libro 31a, exp. 2549, fs. 146-154; y libro 32a, exp. 2636, fs. 108-120.
} 
de este grupo social, se esperaba cubrir los huecos laborales que existían en estos establecimientos y desplazar a los prácticos de farmacia en la elaboración y el despacho de medicinas. En la mente de las autoridades y de los propietarios de farmacia, estaba una idea afín a las creencias acuñadas en torno al trabajo que desarrollaban las profesoras, que a las egresadas de farmacia se les podría ofrecer un salario menor al devengado por los varones; sin embargo, como se expondrá más adelante, estas mujeres no realizaron una actividad laboral acorde con los planes que los primeros tenían en torno a su vinculación con el trabajo farmacéutico.

\section{El comienzo laboral de las farmacéuticas}

Entre 1894 y 1902, lapso en que las mujeres pudieron acceder a la carrera de farmacia en la Facultad de Medicina y Farmacia, sólo tres estudiantes obtuvieron el título de profesoras de farmacia: Elisa Bernal Acosta, Justina Gutiérrez García y María Dolores Navarro.

Estas mujeres no provenían de estratos populares, pero tampoco eran personas de posiciones económicamente desahogadas; las tres procedían de capas medias bajas, donde el nivel socioeconómico familiar les permitió incursionar más allá de la educación secundaria. La ubicación de su posición social ayuda a entender dónde se colocaron para interactuar con las opciones que ofrecía el mercado de trabajo para los farmacéuticos y con las condiciones socioculturales que prevalecían en Guadalajara a principios del siglo xx en torno al trabajo femenino.

A partir de los hechos referidos durante su estancia educativa en la carrera de farmacia y de información vinculada con sus actividades sociales, se puede ubicar relativamente la posición de ellas:

a) Elisa Bernal Acosta fue hija de Francisco Bernal, quien egresó de la Escuela de Medicina y Farmacia en 1862. Su progenitor laboró inicialmente como profesor responsable de algunas farmacias de Guadalajara, ${ }^{13}$ pero después fue propietario de la botica "La Purísima", negocio que también se localizaba en esa ciudad. Ella se crió en un ambiente familiar, donde el contacto cotidiano con la preparación de medicamentos generó una gradual afinidad hacia la farmacia. Su inclinación por ese oficio se vio alentada por el deceso de su padre, ya que esta ausencia precipitó su incorporación al negocio de la botica. Elisa era la hermana mayor de cuatro hermanos; quedó, junto con su madre, a cargo de ese establecimiento. ${ }^{14}$

\footnotetext{
${ }^{13}$ En los primeros meses de 1862, era responsable de la botica de "Nuestra Señora de Belén", situada en contra esquina de la calle de San Diego (El País, diario oficial del gobierno del estado de Jalisco, $1^{\circ}$ de abril de 1862).

${ }^{14} \mathrm{Al}$ igual que Margarita Chorne, primera mujer titulada de la carrera de odontología en México, Elisa Bernal aprendió el oficio de la farmacia en el espacio familiar.
} 
Su familia tenía un patrimonio que le permitió sobrellevar la sobrevivencia diaria, pero no contaba con recursos suficientes para apoyar los estudios de farmacia de la hija mayor. Esta situación llevó a Elisa Bernal a pedir ayuda al director de instrucción pública; el 18 de enero de 1898, le hizo la siguiente petición:

Estoy matriculada para emprender el estudio del primer año de [farmacia], tengo buena conducta y caresco de recursos pecuniarios para emprender y seguir [esos estudios] según consta por las diligencias que acompaño, por lo mismo.

A Ud. C. Director ocurro solicitando una pensión de las que el gobierno del Estado otorga a los estudiantes pobres. ${ }^{15}$

Elisa anexó a esta solicitud una constancia de insolvencia económica avalada por el juez primero de lo civil y de hacienda. Dos días después, el gobernador de la entidad le concedió una pensión mensual para que continuara sus estudios de farmacia, ${ }^{16}$ sin embargo, este apoyo no la liberó de las responsabilidades que compartía con su madre, ya que, en ocasiones no pudo asistir a alguna de las materias debido a que tenía bajo su cargo la atención de la botica familiar. Por ejemplo, en octubre de 1899, se dirigió al director de la Escuela de Medicina y Farmacia para solicitarle derecho a examen en la materia de zoología. En esa demanda, señaló que "me matriculé como alumna de segundo año de farmacia, asistí con regularidad a todas las clases, a excepción de la de Zoología, y esto porque a la hora señalada para dicha cátedra tuve necesidad de ocuparme en un establecimiento de farmacia, propiedad de mi familia, obligación de la que no pude eximirme" ${ }^{17}$

Esta información permite mostrar que Elisa Bernal Acosta provenía de una familia de capas medias bajas que cubría sus necesidades económicas con las utilidades que le otorgaba la botica legada por su padre, negocio que les permitía sobrevivir, pero no les proporcionaba recursos suficientes para sufragar los estudios de farmacia de la hija mayor.

b) De Justina Gutiérrez García, no se dispone de información precisa sobre su origen familiar, pero sí existen datos de su precariedad económica. En un oficio con fecha del 2 de febrero de 1898, remitido al director de la Escuela de Medicina y Farmacia, aparece al margen

Margarita Chorné se inició en la práctica dental a través de su padre, dentista pionero anteriormente dedicado a la orfebrería. En su consultorio, "[...] entrenó en el arte dental a sus hijos Margarita y Rafael, de la misma manera que él había aprendido el oficio de orfebre en el taller de su padre, quien llegó a ser joyero oficial de la Catedral de México. La odontología fue ocupación familiar de los Chorné, quienes ejercieron en el mismo barrio de la ciudad y compartieron los avances del oficio" (Cano, 1996: 72).

${ }^{15}$ AHUG, Fondo Instituciones Educativas de Jalisco, libro 50a, exp. 4865, fs. 181-186.

${ }^{16}$ AHUG, Fondo Instituciones Educativas de Jalisco, libro 50a, exp. 4865, fs. 181-186.

${ }^{17}$ AHUG, Fondo Instituciones Educativas de Jalisco, libro 50a, exp. 4871, fs. 207-214. 
lo siguiente "pago uso de estampillas de a cinco centavos por ser pobre". 18 También se puede decir que vivía condiciones materiales que la empujaban a desempeñar actividades remunerativas. En octubre de 1899, envió una carta al director de esa escuela para pedirle que le concediera derecho a examen ordinario en una materia del segundo año; ella argumentó que había asistido con puntualidad a todas las clases, "con la excepción de la de historia natural, a la que no concurrí por ser empleada en el Liceo de Niñas del Estado". 19

c) En el caso de María Dolores Navarro, no existen muchas evidencias para ubicar su posición social. En el expediente donde se sanciona su inscripción a la carrera de farmacia y su paso por ésta, no se refieren datos que ayuden a precisar su origen social. Lo que sí se puede decir es que provenía de una familia con recursos socioeconómicos que le permitieron estudiar una carrera profesional y le dieron apoyo para establecer una botica.

Como se ve, las familias de estas farmacéuticas no contaban con un vasto patrimonio, pero sí disponían de recursos que facilitaron la promoción de una opción laboral más segura y acorde con los convencionalismos sociales de la época. Las tres instalaron su propia botica, hecho que evitó la "carga moral" asociada a la convivencia con varones en espacios públicos; así, al poco tiempo de su titulación, cada una de ellas inició los trámites para abrir su propio establecimiento.

El 9 de agosto de 1902, Justina Gutiérrez García solicitó al gobernador de la entidad su autorización para abrir una farmacia. Su petición fue remitida al Consejo Superior de Salubridad, organismo que, a los pocos días, aprobó su solicitud. Dada esta certificación, el gobernador le dio permiso para instalar en el cruce de las calles de Santa Teresa y San Cristóbal una botica llamada "La Fe", donde ella trabajó como profesora responsable.20

En octubre de ese mismo año, Elisa Bernal Acosta fue autorizada por ese Consejo para fungir como profesora responsable de la farmacia de "Providencia"; 21 años después, abrió otra denominada "Colonia de Artesanos", ubicada en el cruce de las calles de Mezquitán y Arteaga. $^{22}$

María Dolores Navarro demoró un poco más que sus dos colegas, pero también abrió su propio establecimiento. El 11 de febrero de 1905, el gobernador de Jalisco le concedió el permiso para abrir al servicio público una botica denominada "Nuestra Señora de la Rosa", que se ubicaba en el cruce de las calles de Hidalgo y Cruz Verde.23

\footnotetext{
${ }^{18}$ AHUG, Fondo Instituciones Educativas de Jalisco, libro 50a, exp. 4867, fs. 191-192.

${ }^{19}$ AHUC, Fondo Instituciones Educativas de Jalisco, libro 50a, exp. 4872, fs. 215-222.

${ }^{20} \mathrm{AHJ}$, Jalisco, México, Fondo, F-13, 902, caja 473, exp. 463.

${ }^{21}$ Periódico, El Bien Público, 18 de octubre de 1902.

22 En octubre de 1918, apareció por primera vez como responsable de este establecimiento, pero se desconoce en qué año el Consejo Superior de Salubridad le autorizó la apertura de esta farmacia (El Informador, 12 de octubre de

${ }^{23}$ AHJ, F-13, 905, caja 480, exp. 179.
} 1918). 
Estas tres mujeres concentraron su actividad laboral en la atención del despacho de medicinas a lo largo de las dos primeras décadas del siglo xx; sin embargo, dos de ellas desarrollaron simultáneamente labores que se vincularon con el trabajo de enseñanza. La profesora, Justina Gutiérrez, alternó su rol de farmacéutica con clases en el Liceo de Niñas, en la Escuela Normal Mixta y en la Escuela Comercial e Industrial para Señoritas (ECIS). En la primera escuela, impartió la materia de zoología y botánica, de 1895 a 1902, año en que el gobernador de la entidad decidió cerrar esa institución; ${ }^{24}$ en la segunda, se desempeñó como catedrática de ciencias naturales; y en la tercera. como docente adjunta en materias vinculadas a la carrera de farmacia.

La profesora, Elisa Bernal Acosta, también trabajó en el Liceo de Niñas y en la Escuela Normal Mixta, en las dos instituciones impartió la materia de geografía y cosmografía; 25 después, al igual que Justina Gutiérrez, laboró en la ECIS, donde se desempeñó como docente y encargada de la botica.

Ambas accedieron a estos espacios laborales, gracias a los estudios que realizaron en el Liceo de Niñas y en la carrera de farmacia; sin embargo, su ingreso a estas instituciones no estuvo exento de conflictos, debido a que en la mayoría de las escuelas existieron relaciones de poder que limitaron las posibilidades de acceso a las mujeres a la enseñanza profesional. A principios del siglo XX, la enseñanza de la farmacia, la ingeniería, la jurisprudencia y la medicina era un derecho exclusivo de los hombres -el cual provenía de su predominio absoluto en esas carreras-, que se expresó como "un privilegio natural" del género masculino, sin importar que las opciones educativas fueran de menor escolaridad, como sucedió con las carreras de "segunda clase", creadas para las mujeres en el umbral del siglo Xx.

\section{Las nuevas opciones educativas para las mujeres}

A principios del siglo Xx, el gobernador de Jalisco y coronel, Miguel Ahumada promovió, a semejanza de experiencias similares desarrolladas en el centro del país, ${ }^{26}$ la creación de una escuela de artes y oficios para señoritas, donde las mujeres pudieran obtener conocimientos y habilidades prácticas que "les [asegurara] un trabajo independiente y una vida honrada". Esta iniciativa se concretó en los primeros meses de $1906: 27$ en mayo de ese año, el Congre-

${ }^{24}$ AHJ, IP-5-896 Gua/348.

${ }^{25}$ Biblioteca Pública del Estado de Jalisco, AIP, caja 18, exp. 1-19.

${ }^{26}$ Véase Federico Lazarin (2003), texto en el que describe las opciones de educación técnica que se promovieron en México desde 1871, destacando la creación de la Escuela Nacional de Artes y Oficios para Señoritas, organismo que emergió como un referente para la educación femenina en los estados.

27 Proyecto de ley de la Escuela Comercial e Industrial para Señoritas (AHUG, Fondo Instituciones Educativas de Jalisco, libro 53a, 1921, fs. 56-59). 
so del Estado decretó la creación de una institución de beneficencia que se denominó Escuela Comercial e Industrial para Señoritas (ECIS). ${ }^{28}$ En el documento fundacional, se señaló que su creación obedecía a la necesidad de crear "nuevos horizontes de actividad laboral compatibles con su debilidad corporal", ${ }^{29}$ esta frase muestra, no sólo la presencia de ideas afines a la participación femenina en más espacios laborales, sino también la vigencia de creencias que limitaron el trabajo remunerativo de las mujeres a las "capacidades asociadas a su naturaleza biológica". ${ }^{30}$

La ECIS ofrecía las profesiones de comercio y farmacia a las jóvenes de la sociedad tapatía, ${ }^{31}$ para ingresar a estas opciones sólo se requería acreditar la terminación de los estudios de educación primaria superior; sin embargo, a diferencia de la carrera de farmacia, que existía desde 1839 en Guadalajara, la análoga de la ECIS fue considerada por las autoridades educativas como una opción de menor rango - a las egresadas se les designó como "farmacéuticas de segunda clase".

Su enseñanza comenzó con un cuerpo de profesores integrado por médicos y farmacéuticos varones, destacando en los segundos el catedrático, Adrián Puga. ${ }^{32}$ En este grupo pionero no se incluía a ninguna egresada de farmacia para el trabajo docente en esa escuela:33 sin embargo, al poco tiempo, se invitó a las profesoras Elisa Bernal Acosta y Justina Gutiérrez, quienes desarrollaron labores académicas de apoyo, como la actividad de preparadoras de clase. Este rol subalterno les permitió adquirir mayor reconocimiento, como sucedió con Elisa Bernal, quien logró más aceptación hasta que fue nombrada profesora de la clase de

${ }^{28}$ Decreto núm. 1168, relativo a la creación de la Escuela Comercial e Industrial para Señoritas (AHUc, Fondo Instituciones Educativas de Jalisco, libro 53a, 1921, fs. 62-65).

${ }^{29}$ AHUG, Fondo Instituciones Educativas de Jalisco, libro 53a, 1921, fs. 56-59.

${ }^{30}$ Estas ideas formaban parte de las representaciones construidas durante el porfiriato en torno a las nuevas actividades que empezaban a desarrollar las mujeres. En esa época, como señalan Martínez et al., (2005: 21), se elaboró "un discurso diferenciado, que por un lado exaltaba sus cualidades como madres y esposas, argumento frecuentemente utilizado para las mujeres de clase alta, y por otro, llamaba a los sectores femeninos de clase media y baja a incorporarse al trabajo asalariado".

${ }^{31}$ AHUG, Fondo Miscelánea, libro 6, fs. 94-96.

32 Nació en Cocula el 8 de septiembre de 1858. Estudió la enseñanza preparatoria en el Liceo de Varones del Estado, de 1877 a 1880; y la carrera de farmacia, de 1880 a 182. Presentó su examen profesional en 1884. Este farmacéutico fue reconocido como uno de los profesionistas más capaces de su ramo en Guadalajara a finales del siglo XIX y principios del siguiente; este reconocimiento lo llevó a ocupar puestos públicos vinculados con la higiene y la salubridad, y a participar como catedrático en la Escuela de Medicina y Farmacia, en el Liceo de Varones, en la Escuela Comercial e Industrial para Señoritas y en la Escuela Preparatoria de Jalisco. Murió el 5 de febrero de 1940 en Guadalajara (Gaceta municipal, órgano del Ayuntamiento constitucional de Guadalajara, tomo I, núm. 2, de 15 de febrero de 1917; y El Informador, 6 de febrero de 1940).

${ }^{33}$ A principios de 1907, la directora de la Escuela Comercial e Industrial para Señoritas (ECIS), en el jurado de los exámenes de fin de cursos, propuso a los profesores Justina Gutiérrez y Adrián Puga, para evaluar las materias físicoquímicas (AHUG, Fondo Instituciones Educativas de Jalisco, libro 53a, exp. 4920, fs. 197-198). El 17 de julio de ese año, el gobierno del estado de Jalisco aprobó la lista de sinodales, omitiendo solamente la sugerencia de la profesora, Justina Gutiérrez, quien fue sustituida por el farmacéutico, Elías Figueroa (AHUG, Fondo Instituciones Educativas de Jalisco, libro 53a, fs. 201-202). 
ciencias físico-químicas, hecho que aconteció en julio de 1910, año en el que apareció públicamente como mentora de varias alumnas que realizaron el examen público de esa materia. $^{34}$ Este acceso restringido de las mujeres a la enseñanza de la farmacias se modificó por un suceso que trastocó a la sociedad mexicana a partir de 1910.

\section{La Revolución mexicana y la movilidad laboral de las farmacéuticas}

El 10 de julio de 1914, el ejército constitucionalista hizo su entrada a Guadalajara; a partir de esa fecha, la ciudad se involucró de manera más firme en la convulsión social y política que generó la contienda armada que sacudió al país. El jefe militar de estas fuerzas y general, Manuel M. Diéguez, asumió la dirección política de la entidad, iniciando diversas acciones encaminadas a desplazar a personajes y grupos vinculados con el Partido Católico. En este contexto, se generaron cambios en la enseñanza que abrieron mayores opciones de participación social a las mujeres; la ECIS fue uno de los sitios elegidos para la intervención de esta facción revolucionaria. ${ }^{35}$

A los dos meses de su entronización en el poder político de la entidad, Diéguez impulsó cambios en ese establecimiento. El 31 de agosto de 1914, el director de instrucción pública del estado comunicó a la directora de la ECIS, Edelmira Trejo, que el gobernador, general y comandante militar del estado, Manuel M. Diéguez, dispuso el cese en sus empleos de varios catedráticos en la escuela a su cargo. Entre los profesores suspendidos, se encontraba el farmacéutico, Adrián Puga, ${ }^{36}$ que tenía bajo su responsabilidad las materias de farmacia y química; y Agripina Medina, ${ }^{37}$ quien se desempeñaba como preparadora para las ciencias físico-matemáticas. En su lugar, propusieron a las profesoras de farmacia Elisa Bernal Acosta y Justina Gutiérrez García, quienes impartieron las materias de farmacia y química, respectivamente; ${ }^{38}$ al lado de estas dos farmacéuticas, también se incorporó Dolores de la Torre, ${ }^{39}$ quien fungió como profesora de ciencias físico-químicas. Estas tres mentoras tuvie-

\footnotetext{
${ }^{34}$ Periódico, El Correo de Jalisco, 20 de julio de 1910.
}

35 Fernández (2005: 92) señala que "el gobernador Manuel M. Diéguez (1914-1919) inició reformas anticlericales educativas, laborales y en materia de cultos para establecer alianzas con obreros(as), maestros(as) y campesinos(as) y armar una coalición en contra del fuerte movimiento de acción social que seguía los principios de la encíclica Rerum Novarum (1891). En este contexto revolucionario y anticlerical se buscó incorporar a las mujeres, ya sea como maestras o como madres, al proyecto revolucionario porque jugarían un papel central en la educación de futuros ciudadanos".

36 Se cree que el desplazamiento de Adrián Puga se debió al recelo inicial que las fuerzas constitucionalistas tuvieron hacia las personas que participaron como funcionarios en la administración del Partido Católico en Jalisco. En ese entonces, este farmacéutico formó parte de la mesa directiva del Consejo Superior de Salubridad.

37 Terminó la carrera de farmacia en la ECIS en 1911, año en el que presentó su examen profesional (AHUC, Fondo Instituciones Educativas de Jalisco, libro 5la, exp. 4955, fs. 101-104).

${ }^{38}$ AHUC, Fondo Instituciones Educativas de Jalisco, libro 54a, fs. 311-315.

${ }^{39}$ No egresó de la carrera de farmacia de la ECIS; ella estudió en el Liceo de Niñas, pero se ignora si después ingresó a la Escuela Normal. 
ron como profesora adjunta a Enriqueta Zúñiga, egresada de la carrera de farmacia de esa misma escuela. ${ }^{40}$ Asimismo, al tener bajo su cargo la materia de farmacia, Elisa Bernal Acosta ${ }^{41}$ recibió la responsabilidad de la botica de la ECIS.

Esta intervención del gobierno permitió la incorporación de otras farmacéuticas a la carrera de farmacia, ya que después se agregaron María de la Luz Uribe ${ }^{42}$ y Maura Cortés. ${ }^{43}$ En este cambio de personal, hay un evidente desplazamiento de profesores de farmacia varones, pero también se observa la continuidad de docentes médicos en áreas que no eran competencia de los farmacéuticos. En consonancia con las mentoras mencionadas, se encuentran Pedro L. Alatorre, ${ }^{44}$ a cargo de las materias de fisiología e higiene y elementos de anatomía y terapéutica; y a Francisco $S$. Alatorre, ${ }^{45}$ responsable de las materias de cirugía menor y elementos de patología infantil. ${ }^{46}$

Este rejuego de posiciones, derivado del arribo de las fuerzas constitucionalistas a Guadalajara, permitió a Elisa Bernal Acosta y a Justina Delgado Gutiérrez desplegar durante varios años parte de su trayectoria laboral en la ECIS. Ambas alternaron su actividad docente con la administración de las farmacias de su propiedad; la primera, en la farmacia "Colonia de Artesanos"; y la segunda, en la botica "La Fe", donde se encargaron del despacho de medicinas.

\section{El trabajo de enseñanza de las farmacéuticas}

A partir de septiembre de 1914, Elisa Bernal y Justina Gutiérrez desarrollaron actividades de enseñanza más permanentes y de mayor jerarquía en la ECIs; la primera fue confirmada como profesora de farmacia, y la segunda, recibió el nombramiento de catedrática de química. Las dos tuvieron cierta estabilidad en su trabajo docente durante los tres años siguientes, pero después sobrevinieron algunos cambios que propiciaron una ubicación desigual de ambas.

En los primeros años del gobierno constitucionalista, se promovieron reformas sociales que beneficiaron a las capas populares, sobre todo, a los trabajadores. En esta gradual institucionalización política, Manuel M. Diéguéz erigió lazos con diversas agrupaciones locales, especialmente con organismos liberales, y con personalidades destacadas de los gremios

${ }^{40}$ AHUG, Fondo Instituciones Educativas de Jalisco, libro 54a, fs. 328-329.

${ }^{41}$ En septiembre de 1915, agregó a este nombramiento el cargo de responsable de la botica (AHUG, Fondo Instituciones Educativas de Jalisco, libro 54a, exp. 5315, fs. 362-369).

42 Terminó sus estudios de farmacia en 1912, y se tituló en ese mismo año (AHUC, Fondo Instituciones Educativas de Jalisco, libro 51 A, exp. 4955, fs. 101-104).

${ }^{43}$ Egresó de la carrera de farmacia de la ECIS en 1912, año en el que presentó su examen profesional (AHUG, Fondo Instituciones Educativas de Jalisco, libro 55, exp. 5437, fs. 306-307).

${ }^{44}$ Presentó su examen profesional de medicina en 1887.

${ }^{45}$ Realizó su examen profesional de medicina en 1897.

${ }^{46}$ AHUC, Fondo Instituciones Educativas de Jalisco, libro 54a, fs. 368-369. 
locales. En ese tránsito, amplió las redes de apoyo hacia su persona y creó compromisos con miembros de esas agrupaciones. Como correlato, los integrantes de esas organizaciones recuperaron posiciones dentro de los espacios públicos, como sucedió con algunos directivos de la Sociedad Médico-Farmacéutica de Guadalajara.

Este reacomodo afectó la composición del personal académico de la ECIS. El 14 de octubre de 1917, la Dirección General de Instrucción Pública dispuso que la catedrática Justina Gutiérrez, dejara de impartir la clase de química, para ceder su lugar al profesor de farmacia, Adrián Puga ${ }^{47}$ El regreso de este farmacéutico se vio acompañado por la presencia de los médicos Alberto Onofre Ortega ${ }^{48}$ y Lucas L. Guevara. ${ }^{49}$ En esta recomposición del cuerpo docente, Justina Gutiérrez impartió la clase de ciencias naturales, que después se llamó de biología, en la misma carrera.

Por su parte, Elisa Bernal no se vio afectada por el arribo de varones, porque siguió como profesora de la materia de farmacia y como responsable de la botica hasta mediados de 1919, año en el que se promovieron cambios dentro de la oferta educativa que ofrecía la ECIS. El 28 de junio de ese año, el gobernador decretó el cierre de la carrera de farmacia y dejó sólo la opción de comercio..$^{50}$ Esta modificación generó reacomodos que impactaron de manera desigual en la situación laboral de estas dos profesoras. Así, mientras Elisa Bernal fue designada en septiembre de ese año directora de la ECIS; Justina Gutiérrez dio clases de mecanografía en la carrera de comercio.

El cierre de la carrera de farmacia implicó para la segunda un desplazamiento del trabajo de enseñanza. En septiembre de 1920, Justina Gutiérrez ya no fue ratificada como parte del cuerpo de profesores de esa escuela; en su lugar, se nombró a las farmacéuticas María Trinidad Manjarrez y María Tránsito Mendoza; ${ }^{51}$ sin embargo, esta mujer volvió a tener cierto posicionamiento social con la creación del Departamento de Farmacia ${ }^{52}$ en la Escuela de

${ }^{47}$ En enero de 1917, se desempeñó como inspector de bebidas y comestibles en el municipio de Guadalajara (Gaceta municipal, órgano del Ayuntamiento constitucional de Guadalajara, tomo I, núm. 2 de 15 de febrero de 1917).

${ }^{48}$ Egresó de la Facultad de Medicina y Farmacia en 1915, año en el que presentó su examen profesional.(AHUC, Fondo Instituciones Educativas de Jalisco, libro 37a, fs. 211-212).

49 Nació en Ahualulco del Mercado. Presentó su examen profesional de medicina en 1893, labor que ejerció desde 1895 hasta 1914 (AHJ, F-13, varios años), en este último año, debido a la inestabilidad política existente en las zonas rurales, emigró a Guadalajara, donde empezó a participar en organizaciones liberales que establecieron ligas con el gobierno constitucionalista de Manuel M. Diéguez. Estos vínculos le permitieron el acceso a puestos de representación popular; en noviembre de 1917, apareció propuesto como munícipe propietario por varias agrupaciones liberales para integrar el cabildo de esa ciudad (El Informador, 26 de noviembre de 1917).

${ }^{50}$ Mediante el decreto 1992, el ejecutivo de Jalisco ordenó el cese de la carrera de farmacia en la ECIS (AHUC, Fondo: Instituciones Educativas de Jalisco, libro 50a, exp. 4895, f. 335).

${ }^{51}$ Estudiaron la carrera de farmacia en la ECIS y revalidaron sus estudios en la Escuela de Farmacia en 1923, año en el que ambas realizaron su examen profesional (AHUG, Fondo: Instituciones Educativas de Jalisco, libro 55, exp. 5436, fs. 295-303 y libro 56, exp. 5479, fs. 224-241).

52 Desde mediados de 1921, se creó el Departamento de Farmacia en la Escuela de Medicina y Farmacia, instancia que sirvió para organizar la separación definitiva de la carrera de farmacia de esta dependencia, suceso que ocurrió 
Medicina y Farmacia, ya que fue nombrada secretaria, puesto en el que permaneció desde septiembre de 1921 hasta junio de 1922, pero después de este lapso, a desempeñó labores suplementarias de docencia en la ECIS y la Escuela de Farmacia.

En cambio, a Elisa Bernal el puesto de directora la involucró en eventos públicos asociados con la organización de las actividades escolares, como las fiestas de clausura de fin de cursos y los exámenes profesionales, y le facilitó el acceso a espacios de mayor reconocimiento social dentro del gobierno de la entidad. Ese cargo le permitió acceder a reuniones oficiales con el gobernador de la entidad, donde se decidió la designación de las autoridades de educación primaria ${ }^{53}$ y la aprobación de los programas que se utilizaron en las escuelas de ese nivel educativo. ${ }^{54}$

Su gestión como directora sólo duró dos años, debido a que en septiembre de 1921, el gobernador del estado nombró en su lugar a Amparo Santos, y como secretaria, a s Jesús A. de Curiel. Ella no sólo fue destituida de ese puesto, sino desplazada completamente de la ECIS, porque en la lista propuesta para profesores tampoco apareció asociada a alguna de las asignaturas. ${ }^{55}$ Un mes después, varias alumnas de esa escuela, "considerando la desinteresada y benéfica labor desarrollada en los últimos dos años en la carrera de farmacia", abogaron por su persona ante el gobernador, a quien le pidieron que la designara como profesora del Departamento de Farmacia de la Escuela de Medicina y Farmacia. ${ }^{56}$ Este mandatario contestó, el 24 de octubre de 1921, que "se tomará en cuenta la solicitud de empleo para la señorita profesora Elisa Bernal Acosta, una vez que haya vacante algún puesto en el Departamento que las alumnas indicaron" ${ }^{57}$

Aunque el director de la Escuela de Farmacia propuso a Elisa Bernal Acosta para impartir la cátedra de microbiología en octubre de 1922,58 ya no fue contratada para trabajar en ninguna institución educativa. Este hecho la llevó a concentrarse en el trabajo farmacéutico, retomando con más amplitud la atención y la preparación de medicamentos en la farmacia

en los primeros meses de 1922

${ }^{53}$ El 21 de agosto de 1920, el gobernador interino de Jalisco, Francisco Labastida Izquierdo, reunió en su despacho a directores de planteles vinculados con la educación primaria, donde se incluyó a la profesora, Elisa Bernal Acosta; a inspectores escolares; y a Aurelio Ortega, director de la Escuela Primaria Superior. A todos ellos les pidió que aprobaran la terna que se les presentó para escoger director de educación primaria en el estado, formada por los profesores Manuel R. Alatorre, J. Vicente Negrete y Aurelio Ortega. De esta terna, fue elegido Aurelio Ortega (El Informador, 22 de agosto de 1920).

${ }^{54}$ El 21 de octubre de 1920, la profesora, Elisa Bernal Acosta, participó junto con otros funcionarios en el estudio de los programas que se desarrollaron en las escuelas primarias del estado (El Informador, 21 de octubre de 1920).

55 AHUG, Fondo Instituciones Educativas de Jalisco, libro 57, exp. 5540, f. 448.

${ }^{56}$ AHUG, Fondo Instituciones Educativas de Jalisco, libro 50a, exp. 4904, fs. 419-421.

${ }^{57}$ AHUG, Fondo Instituciones Educativas de Jalisco, libro 50a, exp. 4904, fs. 419-421.

${ }^{58}$ En el expediente, no aparece la respuesta a esta solicitud, pero al costado de ésta se lee, en letras manuscritas, lo siguiente "que dispone el gobernador que la plaza de microbiología quede vacante". AHUC, Fondo Instituciones Educativas de Jalisco, libro 50a, exp. 4922, f. 482). 
"Colonia de Artesanos", ubicada en el Sector Hidalgo, en el cruce de las calles de Mezquitán y J. Palomar. ${ }^{59}$ Esta mujer desarrolló esa labor hasta mediados de noviembre de 1924, ya que el 17 de ese mes se publicó una esquela donde se dio a conocer su fallecimiento; 60 legó a sus hermanas la propiedad de esa farmacia, establecimiento que atendió, principalmente, Refugio Bernal Acosta, a lo largo de los años 20 y parte de los $30 .{ }^{61}$

Por su parte, Justina Gutiérrez continuó con la atención de la farmacia "La Fe" y dejó temporalmente de laborar en la enseñanza a principios de 1926. En esa etapa de suspensión, el gobernador interino de Jalisco, Clemente Sepúlveda, dictó algunas medidas de protección al magisterio, donde destacó la reforma del artículo 99 de la Ley Orgánica de Educación Primaria y Especial, en la que se asentó que "todo maestro a los 30 años de servicio tendría derecho a más de jubilarse, [...] a recibir una pensión vitalicia” (Ibarra y García, 2000: 49). Ella se acogió a esta prerrogativa, porque en abril de ese año solicitó al gobierno de la entidad su jubilación por los servicios prestados en el ramo de instrucción pública durante 30 años. ${ }^{62}$

Esta mujer volvió a trabajar en la docencia, a principios de 1927. En febrero de ese año, fue nombrada profesora interina de farmacognosia y botánica en la Escuela de Farmacia en sustitución del docente, Orestes Corona. ${ }^{63}$ Justina Gutiérrez laboró en este establecimiento hasta el 5 de enero de 1934, fecha en que el rector de la Universidad de Guadalajara informó sobre su cese en el empleo de secretaria y profesora de historia natural, médica y farmacognosia. ${ }^{64}$ Esta actividad fue la última experiencia que tuvo en el campo de la enseñanza, limitándose, a partir de entonces, a preparar y despachar medicinas en la farmacia "La Fe", lugar donde trabajó hasta noviembre de $1941 .{ }^{65}$

María Dolores Navarro emprendió una actividad laboral más discreta; no tuvo otras opciones como sus compañeras, ya que su trayectoria se redujo a la atención y al despacho de medicinas en la farmacia "Nuestra Señora de las Rosas", establecimiento que después se convirtió en la "Botica Occidental", ubicada en el cruce de las calles de Hidalgo y Confederación Revolucionaria.

La revisión de los periódicos locales muestra que esta farmacéutica tuvo dificultades para conservar la licencia de su negocio, ya que en los primeros meses de 1917 trabajó como profesora responsable de la botica de los señores Acosta, labor que dejó de lado para atender su propio despacho. En noviembre de ese mismo año, el Consejo Superior de Salubridad

59, El Informador, 5 de febrero y 23 de septiembre de 1923.

${ }^{60}$ El Informador, 18 de noviembre de 1924.

${ }^{61}$ Desde octubre de 1926, hasta agosto de 1936, la farmacia "Colonia Artesanos" apareció a cargo de Refugio Bernal Acosta (El/nformador, varias fechas que incluyen desde el 17 de octubre de 1926, hasta el 16 de agosto de 1936).

62 Su petición fue abordada por los diputados en la sesión del 6 de mayo de 1926 (El Informador, 7 de mayo de 1926).

${ }^{63}$ AHUG, Fondo Misceláneas, libro 127, fs. 28-39.

${ }^{64}$ AHUG, Fondo Misceláneas, Libro 137, exp. 4-1-540.

${ }^{65}$ El Informador, varias fechas que incluyen desde el 25 de diciembre de 1936, hasta el 2 de noviembre de 1941. 
"le [concederá] permiso para abrir nuevamente al servicio público su botica, con la condición de que siga cumpliendo lo que previene sobre el particular el código sanitario vigente". ${ }^{66}$

Las fuentes permiten afirmar que esta mujer se desempeñó como propietaria y responsable de ese establecimiento hasta el 4 de mayo de 1919:"67 sin embargo, se cree que siguió atendiendo ese negocio porque su nombre apareció publicado en una lista de los médicos y farmacéuticos que tenían registrado su título en la presidencia municipal de Guadalajara, en octubre de $1920 .{ }^{68}$ En los años 20, ya no se encontraron anuncios publicitarios de la "Botica Occidental", pero sí estuvo en la lista de profesores de farmacia registrados en el Departamento de Educación Secundaria y Profesional ${ }^{69}$ y en los registros nacionales del Consejo Superior de Salubridad. ${ }^{70}$ Estos datos Ilevan a suponer que María Dolores Navarro ejerció la profesión de farmacéutica hasta 1929 en Guadalajara.

\section{El estrecho camino de las farmacéuticas}

Los sucesos referidos permiten mostrar que el trayecto laboral de las tres egresadas de farmacia se vio fuertemente influido por el estrecho campo de acción que tenía el gremio farmacéutico a principios del siglo xx en Guadalajara. Ellas, al igual que la mayoría de sus pares, sólo tenían dos opciones: contratarse como profesoras responsables de una botica o abrir su propio establecimiento. La primera opción no era un camino venturoso para las farmacéuticas, porque en ese tiempo compartir un espacio laboral con varones no era bien visto por la sociedad tapatía. La amenaza del escarnio y la maledicencia pública se cernían sobre aquellas mujeres que osaban transitar por senderos considerados como patrimonio exclusivo de los hombres.

Por su parte, la segunda opción no estaba al alcance de la mayoría de los profesores de farmacia, porque la instalación de una botica requería de la disposición de recursos económicos. Esta limitante fue superada por las primeras egresadas, gracias al apoyo familiar y al trabajo que desarrollaban en la enseñanza, como sucedió con Elisa Bernal y Justina Gutiérrez. Las tres abrieron su propio establecimiento, hecho que les permitió establecer una relación de trabajo independiente y una protección contra los señalamientos que la sociedad endosaba a las mujeres que "invadían los espacios masculinos".

${ }^{66}$ El Informador, 23 de noviembre de 1917.

${ }^{67}$ El Informador, varias fechas que incluyen desde el 23 de noviembre de 1917, hasta el 4 de mayo de 1919.

${ }^{68}$ El Informador, 17 de octubre de 1920.

${ }^{69}$ AHUG, Fondo Instituciones Educativas de Jalisco, libro 5la, exp. 4932, fs. 12-14

70 En enero de 1929, María Dolores Navarro apareció en los registros de la Secretaría de Salubridad como farmacéutica que ejerció en Guadalajara (Archivo Histórico de la Secretaría de Salud, Fondo de Salubridad Pública, Sección de ejercicio de la medicina, caja 10, exp. 20). 
Todas procedían de familias que no se ubicaban en situaciones de pobreza, pero sí en posiciones donde se requería su colaboración para sacar a flote la manutención de sus miembros. La vinculación a las actividades remunerativas muestra cómo los apremios económicos las alentaron a superar los obstáculos que limitaban la incursión femenina en ámbitos laborales dominados por los hombres. Las tres también se vieron sobrecogidas por las creencias predominantes en torno a los roles y los límites asignados al género femenino, pero fueron capaces de implementar opciones que les permitieron concretar sus aspiraciones.

Las boticas establecidas por estas mujeres no resolvieron todas sus necesidades económicas, pero fueron espacios que les permitieron realizar sus inquietudes profesionales; sin embargo, la desigual competencia entre sus establecimientos de "medio pelo" con las farmacias de primera clase y las droguerías instaladas por empresarios del ramo motivó a Elisa Bernal y a Justina Gutiérrez a alternar su trabajo farmacéutico con actividades remunerativas en el campo de la enseñanza.

Ambas mujeres iniciaron como profesoras en el Liceo de Niñas, lugar donde no enfrentaron dificultades para desempeñar sus labores. El ambiente preponderantemente femenino les ayudó a desplegar una trayectoria docente sin pormenores, situación que no fue igual al pasar a colaborar en la Escuela Comercial e Industrial para Señoritas. En este lugar, se relacionaron con médicos y farmacéuticos varones que defendieron ese espacio como un usufructo de uso exclusivo para los hombres. Había razones válidas para sostener la presencia de algunos profesores, como sucedió con Adrián Puga, quien era estimado como el profesionista más competente en el campo de la farmacia, pero había otros que no aventajaban en méritos a las mujeres farmacéuticas. A pesar de la preferencia masculina en la enseñanza, estas profesionistas se abrieron camino desde puestos inferiores, como el rol de preparadoras de clase, a través de ese papel subalterno, adquirieron cierto reconocimiento y mayor aceptabilidad social.

\section{Conclusión}

Estas dos mujeres ampliaron sus posibilidades de trabajo, gracias al arribo de las fuerzas constitucionalistas a Guadalajara. La intervención del jefe de esta facción y general, Manuel M. Diéguez, en las instancias educativas, les abrió mayores espacios de enseñanza en la ECIs; sin embargo, su movilidad laboral no se relacionó con la promoción de programas de reivindicación femenina - asunto que apareció hasta los años 20- sino con acciones motivadas por el recelo que los constitucionalistas tuvieron hacia personajes de la entidad, debido a su colaboración como funcionarios dentro de las administraciones estatales a cargo del Partido Católico. 
Durante la Revolución mexicana, vivieron momentos de estabilidad, pero también momentos de incertidumbre, porque las dos resultaron afectadas en sus derechos laborales. Justina Gutiérrez fue desplazada de la enseñanza y relegada a puestos administrativos; y Elisa Bernal, a pesar de que se convirtió la autoridad principal de la ECIS, fue despedida de esa institución. Ambas culminaron su vida laboral con la actividad que describe con mayor precisión el trabajo que principalmente desarrollaban los farmacéuticos en las primeras décadas del siglo xx en Guadalajara: el surtido y la preparación de las medicinas prescritas por los médicos.

Estas mujeres vivían para el trabajo, y su vida social se centró en los espacios donde desarrollaban sus actividades laborales: en la botica, en las escuelas y en el núcleo familiar; no eran hijas de familias pudientes donde la posición económica les permitía departir con miembros de las clases acomodadas, sino de capas bajas, a quienes la necesidad de sobrevivir las llevó a abrir brecha en espacios educativos y laborales dominados por los hombres. Ellas generaron una experiencia que las llevó a disentir de los roles convencionales, sin llegar a radicalismos, porque sus acciones se asociaron más al imperativo de la supervivencia económica, que a una práctica de oposición hacia las estructuras sexistas; sin embargo, su actitud propició implicaciones en su trayectoria de vida porque el modelo de la mujer trabajadora no era compatible con el ideal de la mujer casadera. Al igual que muchas profesoras normalistas, permanecieron solteras a lo largo de su vida.

\section{Fuentes}

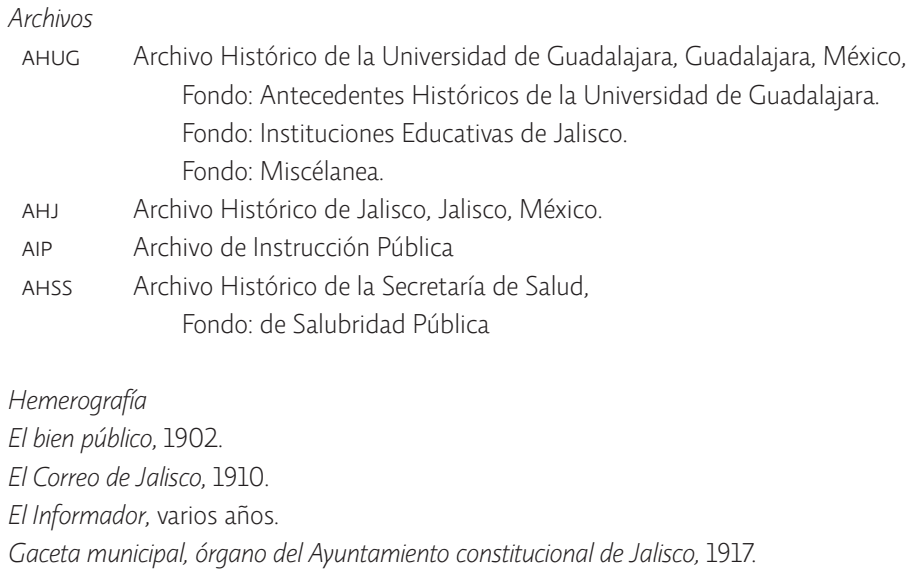


Bibliografía

Alvarado, María de Lourdes (2004), La educación "superior" femenina en el México del siglo Xix. Demanda social y reto gubernamental, unAM-Plaza y Valdés, México.

Bazant, Mílada (coord.) (2013), Biografía. Modelos, métodos y enfoques, El Colegio Mexiquense, A. C., Toluca.

Cano Ortega, Ruth Gabriela (1996), De la Escuela Nacional de Altos Estudios a la Facultad de Filosofía y Letras, 1910-1929. Un Proceso de feminización, tesis para obtener el grado de doctora en historia de México, Facultad de Filosofía y Letras, UNAM, México.

Cárdenas Castillo, Cristina y Martínez, Juan Pío (1997), "Apuntes sobre la formación de profesores en Guadalajara durante la segunda mitad del siglo xIx", Revista Educar, núm. 3, septiembre, pp. 30-37.

Dirección General de Estadística, México (1899), Censo General de la República Mexicana de 1895, México.

Fernández Aceves, María Teresa (1995), "Las mujeres graduadas en la Universidad de Guadalajara: 1925 1933", en Castañeda, Carmen (comp.), Historia social de la Universidad de Guadalajara, CIESASUniversidad de Guadalajara, Guadalajara.

Fernández Aceves, María Teresa (2005), "Debates sobre el ingreso de las mujeres a la universidad y las primeras graduadas en la Universidad de Guadalajara, 1914-1933”, Revista La Ventana, núm. 21, pp. 90-106.

Figueroa Gómez, Yuriria (2009), Escolarizar lo femenino: discursos, proyectos y realidades en torno a la educación superior de la mujer en Guadalajara durante la segunda mitad del siglo XIX, tesis de maestría en investigación en ciencias de la educación, Universidad de Guadalajara, Guadalajara, Jalisco.

García Alcaraz, María Guadalupe (2005), "Las maestras tapatías: celibato y disciplina. (1867-1910)", en Instituto Superior de Investigación y Docencia para el Magisterio (ISIDM), Maestros: historicidad, cotidianidad y proyectos, ISIDM-Editorial Amate, Zapopan, pp. 13-24.

Ibarra Ibarra, Sonia y Oscar García Carmona (2000), Ochenta años de educación pública federal en Jalisco, tomo I, SNTE, Guadalajara.

Lazarín Miranda, Federico (2003), "Enseñanzas propias de su sexo. La educación técnica de la mujer, 1871-1934", en María Adelina Arredondo (coord.), Obedecer, servir y resistir. La educación de las mujeres en la historia de México, uPN-Porrúa, México, pp. 249-277.

Martínez, Sandra, Patricia Aceves y Alba Morales (2005), "Esther Luque Muñoz: primera farmacéutica de la Escuela Nacional de Medicina", Revista Mexicana de Ciencias Farmacéuticas, vol. 36, núm. 004, octubre-diciembre, pp. 20-27.

Mata Vargas, E. (1985), El Colegio de San Diego, Departamento de Educación Pública del Estado de Jalisco, Colección de breviarios, serie Monografías 1, Guadalajara, México.

Núñez, Patricia (1994), La enseñanza media en Jalisco (siglo XIX), El Colegio de Jalisco-SEP, Zapopan.

Orendain, Leopoldo I. (1969), Cosas de viejos papeles, Banco Industrial de Jalisco, Guadalajara.

Oropeza Sandoval Luciano (2016), "Las argucias de las mujeres para ingresar a los espacios públicos: las primeras estudiantes de farmacia en Guadalajara", en Laura Catalina Díaz Robles, Anayanci Fregoso Centeno y María Guadalupe García Alcaraz (coords.) Mujeres, niños y niñas en la historia. América Latina, siglos xix y xx, Universidad de Guadalajara, Guadalajara, pp. 289-311.

Peregrina, Angélica (2006), "La carrera magisterial: una opción para las mujeres de Guadalajara (19001925)", Sinéctica, núm. 28, febrero-julio, pp. 17-27.

Stone, Lawrence (1986), El pasado y el presente, Fondo de Cultura Económica, México. 
Luciano Oropeza Sandoval. Doctor en ciencias de la educación y profesor-investigador titular del Departamento de Estudios en Educación de la Universidad de Guadalajara. Sus líneas de investigación son historia de la educación e historia de las profesiones, siglos XIX y xx. Entre sus publicaciones recientes se encuentran García Alcaraz, María Guadalupe y Luciano Oropeza Sandoval (2016), "Universidad de Guadalajara y regionalidad: un proyecto educativo para el occidente y noroeste de México", en Revista Diálogos sobre educación, número 11, Departamento de Estudios en Educación, Guadalajara, enero, pp. 1-11; y Luciano Oropeza Sandoval (2016), "Las egresadas de trabajo social y la regionalidad", en Martha Valadez Huizar, María Guadalupe Moreno Bayardo y Cándida Elizabeth Vivero Marín, Avances y perspectivas de la investigación educativa y de género, Universidad de Guadalajara, Guadalajara, México, pp. 79-94."Las argucias de las mujeres para ingresar a los espacios públicos: las primeras estudiantes de farmacia en Guadalajara”, en Laura Catalina Díaz Robles, Anayanci Fregoso Centeno y María Guadalupe García Alcaraz. Mujeres, niños y niñas en la historia. América Latina, siglos XIX y Xx, Universidad de Guadalajara, Guadalajara, México, pp. 289-311.

Recibido: 15 de enero de 2017.

Aceptado: 23 de marzo de 2017. 